\title{
Screw dislocation in a Bi-medium within strain gradient elasticity revisited
}

https://doi.org/10.1515/jmbm-2019-0008

Received Apr 8, 2019; accepted Jul 8, 2019

\begin{abstract}
In this paper, we consider a straight screw dislocation near a flat interface between two elastic media in the framework of strain gradient elasticity (as studied by Gutkin et. al. [1]) by now taking care of some incomplete calculations). Closed form solutions for stress components and the Peach-Koehler force on the dislocation have been derived. It is shown that the singularities of the stress components at the dislocation line are eliminated and both components are continuous and smooth across the interface. The effect of the distance of the dislocation position from the interface on the maximum value of stress is investigated. Unlike in the case of classical solution, the image force remains finite when the dislocation approaches the interface. It is shown that the dislocation is attracted by the medium with smaller shear modulus or smaller gradient coefficient.
\end{abstract}

Keywords: Strain gradient elasticity; Screw dislocation; Bi-medium; Image force

\section{Introduction}

The study of the elastic interaction of dislocations and inclusions is of considerable importance for understanding the strengthening and hardening mechanism of crystalline materials, especially composite materials $[2,3]$. Several investigations have been conducted to assess dislocation-inclusion interaction of a straight dislocation with a semi-infinite interface between two dissimilar media $[1,4-7]$; interactions of edge and screw dislocations

\footnotetext{
*Corresponding Author: Kamyar M. Davoudi: School of Engineering and Applied Science, Harvard University, Cambridge, MA 02138, USA

(Current Address): Faculty of Engineering, University of Alberta, Edmonton, AB, Canada E-mail: davoudi@alumni.harvard.edu, kdavoudi@ualberta.ca

Elias C. Aifantis: School of Engineering, Aristotle University of Thessaloniki, 54006 Thessaloníki, Greece
}

with a circular inclusion [8-11]; and the interaction of dislocations with coated fibers [12-16]. Most of these attempts have been made in the context of conventional or classical elasticity. However, classical elasticity solutions are characterized by singularities in the components of the stress and strain fields. In addition, the force acting on the dislocation due to the existing interface becomes infinite as the dislocation approaches the interface, and some components of the stress field experience abrupt jumps at the interface. Gutkin et. al. [1, 7] indicated that these jumps can be justified only from a macroscopic point of view, and these solutions are inadmissible from a nanoscopic point of view. Thus, classical elasticity breaks down at the dislocation core and at the interface.

Additionally, it has been experimentally and computationally shown that elastic (see, e.g., [17-21]) and plastic (see, e.g., [21-30]) responses of materials at small length scales can be size dependent. Classical continuum theories are, however, scale-free and, hence, they cannot predict the behavior of materials at very small scales. In order to remedy this critical shortcoming, one or more material length scales are incorporated into the continuum constitutive equations. One of these fortified continuum theories is strain gradient elasticity where strain energy density or Hooke's law contains gradients of elastic strain and/or stress fields.

The constitutive equation of a simple theory of strain gradient elasticity proposed by the second author and coworkers (for a recent review see [31] and refs quoted therein) reads

$$
\left(1-l^{2} \nabla^{2}\right) \sigma=\left(1-c^{2} \nabla^{2}\right)[\lambda(\operatorname{tr} \epsilon) 1+2 \mu \epsilon],
$$

where $\epsilon$ and $\sigma$ denote the elastic stress and strain tensors, $\lambda$ and $\mu$ are the usual Lamé constants, 1 the unit tensor, $\nabla^{2}$ the Laplacian, and $l$ and $c$ are two different gradient coefficients with the dimension of length. The stress and strain gradients are added to dispense the singularity of the stress and strain at the dislocation core and the crack tips. In analogy with what is now commonly known as the Ru-Aifantis theorem [31], a simple approach to solve boundary-value problems (BVPs) associated with Eq. (1) is to use existing solutions of classical elasticity for the same (traction) BVP. In fact, providing that appropriate care is 
taken for extra boundary conditions (on account of the higher order terms) or conditions at infinity, $u$ and $\sigma$ can be found through the inhomogenous Helmhotz equations

$$
\begin{aligned}
& \left(1-c^{2} \nabla^{2}\right) u=u^{0}, \\
& \left(1-l^{2} \nabla^{2}\right) \sigma=\sigma^{0},
\end{aligned}
$$

where $u^{0}$ and $\sigma^{0}$ are the solutions of the same BVP in classical elasticity. Eqs. (2) have been successfully applied to study the interaction between a dislocation and an interface $[1,7,11,32-34]$.

In this paper, we use the theory of strain gradient elasticity described by Eq. (1) to study the interaction between a straight screw dislocation and a flat interface. The same problem was studied by Gutkin et. al. [1] with some deficiencies. For example, the stress components were not smooth or continuous despite the additional boundary conditions they imposed. In addition, it was not explained why the behavior of screw and edge dislocations near the interface of two materials with different gradient coefficients are different. These motivated us to reconsider this problem and calculate the stresses and the image force acting on the dislocation.

\section{Classical solution}

Consider two elastic isotropic, perfectly bonded semiinfinite bodies denoted by region $1(x \geq 0)$ and region 2 $(x \leq 0)$ with different Lamé coefficients and gradient constants. Such a solid is called a bi-medium. Super- and subscripts 1 and 2 are exclusively used for reference to these two regions and the omission of super- or sub-script indicates that the relationship is true for both regions. Suppose a straight screw dislocation with the Burgers vector $b=(0,0, b)$ is situated in region 1 on the $x$-axis at $x=x_{0}$, and the dislocation line is parallel to the interface of the media (Fig. 1).

In classical elasticity, the $z x$-component of the stress field and the $z$-component of the displacement field due to the interaction of the screw dislocation and the interface should be continuous across the interface $(x=0)$. Imposing these boundary conditions, the classical stresses (in the units of $\mu_{1} b / 2 \pi$ ) are given by Head [4] as

$$
\begin{array}{lll}
\sigma_{z x}^{O(1)}=-\frac{y}{r_{1}^{2}}+\gamma \frac{y}{r_{2}^{2}}, & \sigma_{z x}^{O(2)}=-(1+\gamma) \frac{y}{r_{1}^{2}}, \\
\sigma_{z y}^{O(1)}=\frac{x-x_{0}}{r_{1}^{2}}+\gamma \frac{x+x_{0}}{r_{2}^{2}}, & \sigma_{z y}^{O(2)}=(1+\gamma) \frac{x-x_{0}}{r_{1}^{2}},
\end{array}
$$

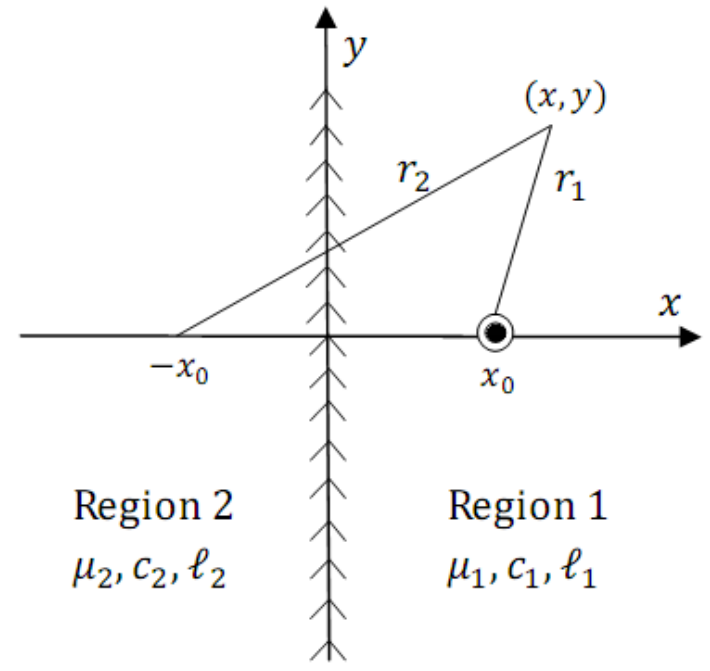

Figure 1: A screw dislocation near a bi-medium interface

where $r_{1}=\sqrt{\left(x-x_{0}\right)^{2}+y^{2}}, r_{2}=\sqrt{\left(x+x_{0}\right)^{2}+y^{2}}$ as depicted in Fig. 1 and $\gamma$ is defined by

$$
\gamma=\frac{\mu_{2}-\mu_{1}}{\mu_{2}+\mu_{1}}
$$

As indicated earlier, the $z x$-component of the classical stress field should be continuous on $x=0$, while the component $\sigma_{z y}$ has an abrupt jump across the interface:

$$
\sigma_{z y}^{0(1)}(x=0, y)-\sigma_{z y}^{0(2)}(x=0, y)=2 \gamma \frac{x_{0}}{x_{0}^{2}+y^{2}} .
$$

It is worthwhile to note that this jump goes to infinity when the dislocation nears the interface. Since the $z y$ component of the stress field does not contribute to the traction vector, this jump is justified in classical elasticity. Gutkin et al. [1, 7] indicate that this jump is unphysical and the nature of it is quite unclear in nanoscopic point of view: In fact, the jump in $z y$-component is a consequence of the approximation of classical continuum models, which may become insufficient for describing nanoscale phenomena $[1,7]$. It may, thus, be desirable for the interface stress jump to be eliminated from the solution of this problem within any generalized theory of elasticity aiming to consider nanoscale phenomena.

\section{Gradient solution}

Let us consider the same problem within the theory of strain gradient elasticity. Equations (2) must be solved for both regions 1 and 2. Also as mentioned in section 2, due to the presence of higher gradient terms, prescription of extra boundary conditions is required. 
To find the $z x$-component of the stress field, it is convenient to decompose it into a particular part, $\sigma_{z x}^{p(j)}(j=1,2)$ and the homogeneous part, $\sigma_{z x}^{h(j)}$. It can easily be shown that

$$
\begin{aligned}
\sigma_{z \chi}^{p(1)} & =\sigma_{z \chi}^{0(1)}+\frac{y}{l_{1} r_{1}} K_{1}\left(\frac{r_{1}}{l_{1}}\right), \\
\sigma_{z x}^{p(2)} & =\sigma_{z \chi}^{0(2)},
\end{aligned}
$$

where $\sigma_{z x}^{O(1)}$ and $\sigma_{z x}^{O(2)}$ are the classical solutions given by Eq. (3). Using the Fourier transform with respect to $y$

$$
\begin{aligned}
\hat{\sigma}_{z x}^{h(j)}(x, s) & =F\left\{\sigma_{z x}^{h(j)}(x, y) ; y \rightarrow s\right\} \\
& =\frac{1}{\sqrt{2 \pi}} \int_{-\infty}^{\infty} \sigma_{z x}^{h(j)}(x, y) e^{-i s y} d y,
\end{aligned}
$$

the corresponding equation of the homogeneous part is reduced to the following ordinary differential equation

$$
\left(1-l^{2} s^{2}-l^{2} \frac{d^{2}}{d x^{2}}\right) \hat{\sigma}_{z x}^{h(j)}=0 .
$$

Considering the fact that the stress components approach zero as $x$ approaches $\infty$ or $-\infty$, and using the inverse of Fourier transform, the homogenous solutions read as follows

$$
\begin{aligned}
& \sigma_{z x}^{h(1)}=\frac{1}{\sqrt{2 \pi}} \int_{-\infty}^{\infty} A(s) e^{-x \sqrt{s^{2}+\frac{1}{l_{1}^{2}}}} e^{i s y} d s, \\
& \sigma_{z x}^{h(2)}=\frac{1}{\sqrt{2 \pi}} \int_{-\infty}^{\infty} a(s) e^{x \sqrt{s^{2}+\frac{1}{l_{2}^{2}}}} e^{i s y} d s,
\end{aligned}
$$

where $A(s)$ and $a(s)$ are to be determined by the boundary conditions proposed and used by Gutkin et al. [7]:

$$
\begin{aligned}
& \sigma_{z x}^{(1)}(x=0, y)=\sigma_{z x}^{(2)}(x=0, y), \\
& \frac{\partial \sigma_{z x}^{(1)}}{\partial x}(x=0, y)=\frac{\partial \sigma_{z x}^{(2)}}{\partial x}(x=0, y) .
\end{aligned}
$$

The above boundary conditions provide not only a continuous but also a smooth transition of the profile of $\sigma_{z x}$ across the interface. If $\hat{\sigma}_{z x}^{(j)}(x, s)=F\left\{\sigma_{z x}^{(j)}(x, y) ; y \rightarrow s\right\}$, the above boundary conditions will be equivalent to

$$
\begin{aligned}
& \hat{\sigma}_{z x}^{(1)}(x=0, s)=\hat{\sigma}_{z x}^{(2)}(x=0, s) . \\
& \frac{\partial \hat{\sigma}_{z x}^{(1)}}{\partial x}(x=0, s)=\frac{\partial \hat{\sigma}_{z x}^{(2)}}{\partial x}(x=0, s) .
\end{aligned}
$$

The Fourier transform of gradient solutions are

$$
\begin{aligned}
& \hat{\sigma}_{z x}^{(1)}=-i \sqrt{\frac{\pi}{2}} \operatorname{sgn}(s)\left(e^{-\left|s\left(x-x_{0}\right)\right|}-\gamma e^{-\left|s\left(x+x_{0}\right)\right|}\right) \\
& +i \sqrt{\frac{\pi}{2}} \frac{s}{\lambda_{1}} e^{-\left|x-x_{0}\right| \lambda_{1}}+A(s) e^{-\chi \lambda_{1}},
\end{aligned}
$$

and

$$
\hat{\sigma}_{z x}^{(2)}=-(1+\gamma) i \sqrt{\frac{\pi}{2}} \operatorname{sgn}(s) e^{-\left|s\left(x-x_{0}\right)\right|}+a(s) e^{x \lambda_{2}},
$$

where $\lambda_{j}=\sqrt{s^{2}+\frac{1}{l_{j}^{2}}}$. Substitution of $\hat{\sigma}_{z x}^{(1)}$ and $\hat{\sigma}_{z x}^{(2)}$ into Eqs. (11) gives us the unknown functions $A(s)$ and $a(s)$. After some simplifications, we obtain

$$
\begin{aligned}
& \sigma_{z x}^{(1)}=\sigma_{z x}^{0(1)}+\frac{y}{l_{1} r_{1}} K_{1}\left(\frac{r_{1}}{l_{1}}\right) \\
& +\int_{0}^{\infty} \frac{\sin (s y)}{\lambda_{1}+\lambda_{2}} e^{-x \lambda_{1}}\left[\frac{\lambda_{1}-\lambda_{2}}{\lambda_{1}} s e^{-x_{0} \lambda_{1}}-2 \gamma \lambda_{2} e^{-x_{0} s}\right] d s, \\
& \sigma_{z x}^{(2)}=\sigma_{z x}^{0(2)}+2 \int_{0}^{\infty} \frac{\sin (s y)}{\lambda_{1}+\lambda_{2}} e^{x \lambda_{2}}\left[s e^{-x_{0} \lambda_{1}}+2 \gamma \lambda_{1} e^{-x_{0} s}\right] d s .
\end{aligned}
$$

Figure 2 compares the classical and gradient solutions of the $z x$-component of the stress field. Both $\sigma_{z x}^{0}\left(x, y=2 l_{1}\right)$ and $\sigma_{z x}\left(x, y=2 l_{1}\right)$ are continuous across the interface. Unlike $\sigma_{z x}^{0}\left(x, y=2 l_{1}\right), \sigma_{z x}\left(x, y=2 l_{1}\right)$ is also smooth at the interface.

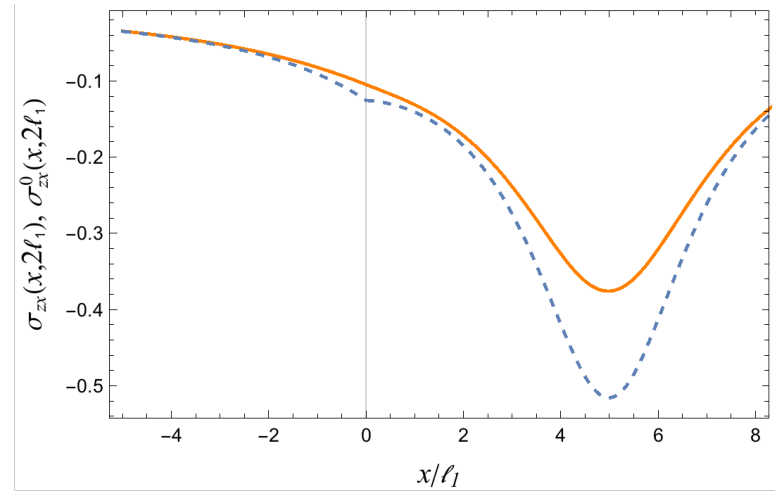

Figure 2: The profile of $\sigma_{z x}(x, y=2)$ when $\mu_{2} / \mu_{1}=10$ and $l_{2} / l_{1}=2$ with the dislocation being placed at $x_{0} / l_{1}=5$. Solid and dashed curves correspond to the gradient and classical solutions, respectively. The stress values are given in units of $\mu_{1} b /\left(2 \pi l_{1}\right)$.

In the same manner, the gradient solution of the $z y$ component of the stress field can be obtained

$$
\begin{aligned}
& \sigma_{z y}^{(1)}=\sigma_{z y}^{0(1)}-\frac{x-x_{0}}{l_{1} r_{1}} K_{1}\left(\frac{r_{1}}{l_{1}}\right)+\frac{1}{\sqrt{2 \pi}} \int_{-\infty}^{\infty} D(s) e^{-x \lambda_{1}} e^{i s y} d s, \\
& \sigma_{z y}^{(2)}=\sigma_{z y}^{0(2)}+\frac{1}{\sqrt{2 \pi}} \int_{-\infty}^{\infty} d(s) e^{x \lambda_{2}} e^{i s y} d s
\end{aligned}
$$


The unknown functions, $D(s)$ and $d(s)$, can be given by imposing the following boundary conditions

$$
\begin{aligned}
& \sigma_{z y}^{(1)}(x=0, y)=\sigma_{z y}^{(2)}(x=0, y), \\
& \frac{\partial \sigma_{z y}^{(1)}}{\partial x}(x=0, y)=\frac{\partial \sigma_{z y}^{(2)}}{\partial x}(x=0, y),
\end{aligned}
$$

or in terms of their Fourier transforms

$$
\begin{aligned}
& \hat{\sigma}_{z y}^{(1)}(x=0, s)=\hat{\sigma}_{z y}^{(2)}(x=0, s), \\
& \frac{\partial \hat{\sigma}_{z y}^{(1)}}{\partial x}(x=0, s)=\frac{\partial \hat{\sigma}_{z y}^{(2)}}{\partial x}(x=0, s),
\end{aligned}
$$

where $\hat{\sigma}_{z y}^{(j)}(x, s)=F\left\{\sigma_{z y}^{(j)}(x, y) ; y \rightarrow s\right\}$. After some simplifications, the final results turn out

$$
\begin{aligned}
& \sigma_{z y}^{(1)}=\sigma_{z y}^{0(1)}-\frac{x-x_{0}}{l_{1} r_{1}} K_{1}\left(\frac{r_{1}}{l_{1}}\right) \\
& +\int_{0}^{\infty} \frac{\lambda_{2} \cos (s y)}{\lambda_{1}+\lambda_{2}} e^{-\chi \lambda_{1}}\left[\frac{\lambda_{1}-\lambda_{2}}{\lambda_{2}} e^{-x_{0} \lambda_{1}}-2 \gamma e^{-x_{0} s}\right] d s, \\
& \sigma_{z y}^{(2)}=\sigma_{z y}^{0(2)}+2 \int_{0}^{\infty} \frac{\lambda_{1} \cos (s y)}{\lambda_{1}+\lambda_{2}} e^{x \lambda_{2}}\left[e^{-x_{0} \lambda_{1}}+\gamma e^{-x_{0} s}\right] d s .
\end{aligned}
$$

Figure 3 shows that while the gradient term for the $z y$ component of the stress field is continuous and smooth across the interface, the value of the classical stress jumps on the interface.

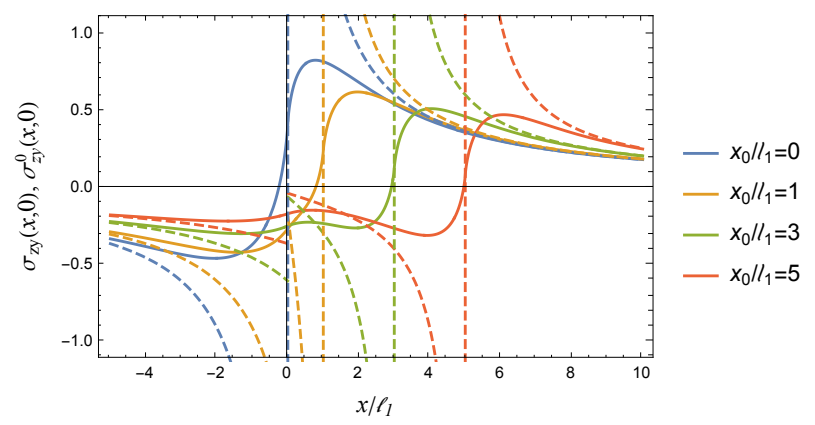

Figure 3: The profile of $\sigma_{z y}(x, y=0)$ when $\mu_{2} / \mu_{1}=10, l_{2} / l_{1}=2$, and the dislocation is placed at different locations $x_{0} / l_{1}$. Solid and dashed curves are pertinent to the gradient and classical solutions, respectively. The stress values are given in units of $\mu_{1} b /\left(2 \pi l_{1}\right)$. the inner structure of both regions on the maximum stress magnitude, $\max \left|\sigma_{z y}\right|$. In the case that materials 1 and 2 have different shear moduli, but the same gradient coefficients, numerical evaluation of $\max \left|\sigma_{z y}\right|$ shows that when the material 1 is elastically softer than material 2 (when $x_{0} \geq 0$ ), $\max \left|\sigma_{z y}\right|$ increases when the dislocation is shifted towards the interface. In this case, the peak value of stress is obtained when the dislocation is situated on the interface. If material 2 is elastically harder, the value of $\max \left|\sigma_{z y}\right|$ starts increasing when the dislocation is shifted toward the interface and starts decreasing when the dislocation is closer than $\approx 3 l$. These facts are shown in Fig. 4 .

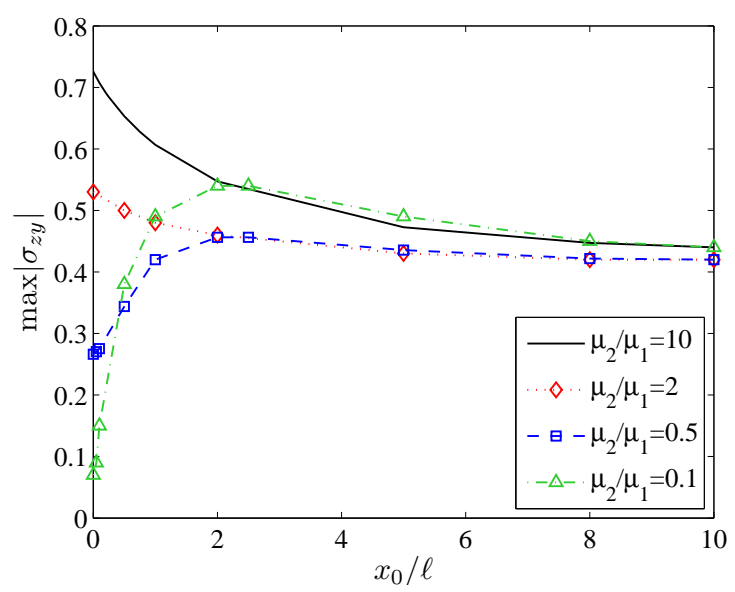

Figure 4: Dependence of $\max \left|\sigma_{z y}\right|$ on the normalized dislocation position $x /$ lfor $l_{1}=l_{2}=$ land different values of $\mu_{2} / \mu_{1}$. The stress values are given in units of $\mu_{1} b /\left(2 \pi l_{1}\right)$.

If the regions have the same elastic constants, but different gradient coefficients, when $l_{2} / l_{1}<1, \max \left|\sigma_{z y}\right|$ increases with the decreasing ratio and when $l_{2} / l_{1}>1$, this trend reverses. These results agree with what Davoudi et al. $[11,33]$ have obtained for a screw dislocation inside or outside a circular inhomogeneity.

\section{Image force}

Now, let us consider the image force (Peach-Koehler force) $F_{X}$ per unit length of the dislocation imposed by the inter-

\section{Size effect}

An advantage of the gradient solution as compared to the classical solution is that one can investigate the effect of 


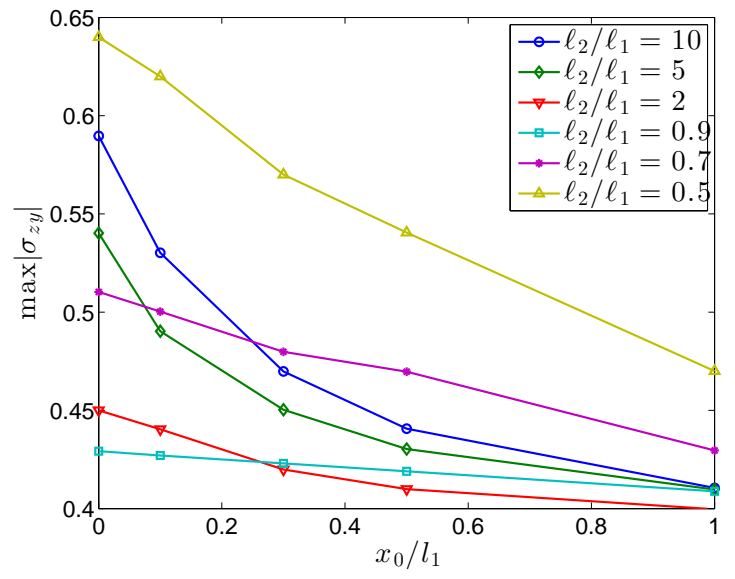

Figure 5: Dependence of $\max \left|\sigma_{z y}\right|$ on the normalized dislocation position $x / l_{1}$ for $\mu_{1}=\mu_{2}$ and different values of $l_{2} / l_{1}$. The stress values are given in units of $\mu_{1} b /\left(2 \pi l_{1}\right)$.

face. The gradient solution (in units of $\mu_{1} b^{2} / 2 \pi$ ) reads

$$
\begin{aligned}
& F_{x}=b \sigma_{z y}^{(1)}\left(x=x_{0}, y=0\right)=\frac{\gamma}{2 x_{0}} \\
& +\int_{0}^{\infty} \frac{\lambda_{2}}{\lambda_{1}+\lambda_{2}}\left[\frac{\lambda_{1}-\lambda_{2}}{\lambda_{2}} e^{-2 x_{0} \lambda_{1}}-2 \gamma e^{-x_{0}\left(s+\lambda_{1}\right)}\right] d s,
\end{aligned}
$$

in which the first term on the right-hand side forms the classical solution and the integral term comes from the gradient solution. It is evident that the classical image force becomes infinite as the dislocation nears the interface $\left(x_{0} \rightarrow 0\right)$.

The sign of $F_{X}$ determines whether the dislocation is repelled or attracted toward the interface. Since $x_{0} \geq 0$, the positive value of $F_{X}$ means attraction and the negative value of it indicates repulsion.

For a purely elastic interface $\left(\mu_{1} \neq \mu_{2}, l_{1}=l_{2}\right)$, the formula of the image force, $F_{X}^{e l}=F_{X}$, is simplified to

$$
F_{x}^{e l}=\frac{\gamma}{2 x_{0}}-\gamma \int_{0}^{\infty} e^{-x_{0}(s+\lambda)} d s, \quad \lambda_{1}=\lambda_{2}=\lambda .
$$

The numerical evaluation of $F_{x}^{e l}$ is depicted in Fig. 6a. It is seen that when $\mu_{2}>\mu_{1}, F_{X}^{e l}$ is positive and when $\mu_{2}<\mu_{1}, F_{x}^{e l}$ becomes negative. This means that the dislocation is pushed away by the harder medium. The maximum force on the dislocation occurs when the dislocation is at $x_{0} \approx l$.

In the case of a purely gradient interface $\left(\mu_{1}=\mu_{2}, l_{1}=\right.$ $l_{2}$ ), the image force, $F_{x}^{g r}=F_{x}$ reduces to

$$
F_{X}^{g r}=\int_{0}^{\infty} \frac{\lambda_{1}-\lambda_{2}}{\lambda_{1}+\lambda_{2}} e^{-2 x_{0} \lambda_{1}} d s
$$

In the case of $l_{1}>l_{2}$ or $\lambda_{1}<\lambda_{2}$, the gradient image force is negative and in the case of $l_{1}<l_{2}$ or $\lambda_{1}>\lambda_{2}$, the gradient image force has a positive value (Fig. 6b). In other words, the dislocation is pulled into the medium having a smaller gradient coefficient. This result agrees with what Mikaelyan et. al. [7] obtained for an edge dislocation in a bi-medium. Since Gutkin et. al. [1] made certain miscalculations in obtaining the gradient solution for a screw dislocation in a bi-medium, their results differ from the result obtained by Mikaelyan et. al. [7] for an edge dislocation in a bi-medium; the nature of this difference could not be determined.
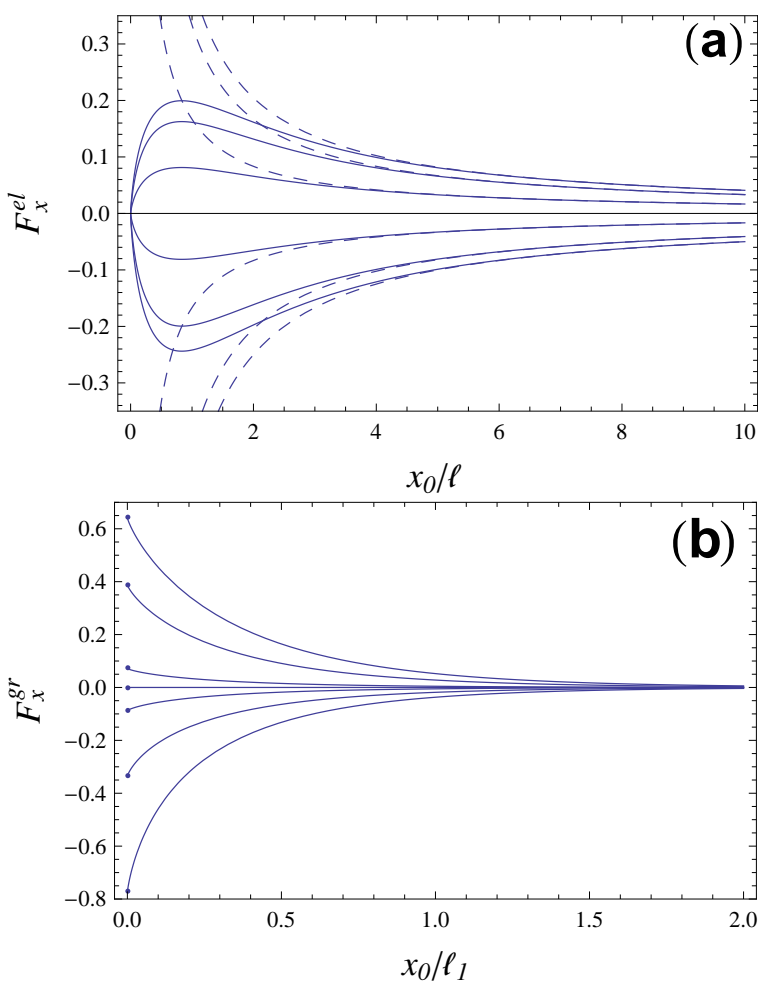

Figure 6: Dependence of the image forces (a) $F_{x}^{e l}$ and (b) $F_{x}^{g r}$ on the normalized positions, (a) $x_{0} / \ell$ and (b) $x_{0} / \ell_{1}$, of a screw dislocation near (a) a purely elastic interface for $\ell_{1}=\ell_{2}=\ell$ and $=10,5,2,0.5$, 0.1 , and 0 (from top to bottom); (b) a purely gradient interface with $\mu_{2}=\mu_{1}$ and $\ell_{2} / \ell_{1}=10,2,1.1,1,0.9,0.7$ and 0.5 . The force values are given in units of (a) $\mu_{1} b^{2} /(2 \pi \ell)$ and (b). The dashed curves in (a) correspond to the classical solutions.

\section{Conclusions}

In this paper, we have employed gradient elasticity theory to calculate the nonsingular stress components are not singular at the dislocation line which also experiences a continuous and smooth transition at the interface. The classi- 
cal and gradient stress fields coincide at a distance larger than $\sim 5-7 l$ from the dislocation line or the interface.

For a purely elastic interface, when $\mu_{2}>\mu_{1}$, the maximum shear stress in the media, $\max \left|\sigma_{z y}\right|$, monotonically increases as the dislocation approaches the interface. When $\mu_{2}<\mu_{1}$, however, $\max \left|\sigma_{z y}\right|$ reaches its peak value when the dislocation is a few l's away from the interface. For a purely gradient interface, $\max \left|\sigma_{z y}\right|$ attains its peak value when the dislocation is at the interface.

The force on the dislocation remains finite no matter where the dislocation is, unlike the classical force on the dislocation which approaches infinity as the dislocation approaches the interface. It is shown that the dislocation is pulled into the medium of smaller shear stress for a purely elastic interface and of smaller gradient coefficient for a purely gradient interface.

\section{References}

[1] Gutkin M.Y., Mikaelyan K.N., Aifantis E.C., Screw dislocation near interface in gradient elasticity, Scr. Mater. 2000, 43(6), 477.

[2] Haasen P., Physical Metallurgy, 1996, 3rd ed., Cambridge University Press.

[3] Hull D., Bacon D.J., Introduction to Dislocations, 2011, 5th ed. Elsevier.

[4] Head A.K., The Interaction of Dislocations and Boundaries, London, Edinburgh, Dublin Philos. Mag. J. Sci., 1953, 44(348), 92.

[5] Head A.K., Edge Dislocations in Inhomogeneous Media, Proc. Phys. Soc. Sect. B, 1953, 66(9), 793.

[6] Gao F., Screw dislocation in a bi-medium in non-local elasticity, J. Phys. D Appl. Phys., 1990, 23(3), 328.

[7] Mikaelyan K.N., Gutkin M.Y., Aifantis E.C., Edge dislocations near phase boundaries in the gradient theory of elasticity, Phys. Solid State, 2000, 42(9),1659.

[8] Dundurs J., Mura T.J., Interaction between an edge dislocation and a circular inclusion, Mech. Phys. Solids, 1964, 12(3), 177.

[9] Dundurs J., Sendeckyj G., Edge dislocation inside a circular inclusion, J. Mech. Phys. Solids, 1965, 13(3),141.

[10] Pan K., Interaction of a dislocation and an inclusion in nonlocal elasticity, Int. J. Eng. Sci., 1996, 34(14),1675.

[11] Davoudi K.M., Gutkin M.Y., Shodja H.M., A screw dislocation near a circular nano-inhomogeneity in gradient elasticity, Int. J. Solids Struct., 2010, 47(6), 741.

[12] Xiao Z.M., Chen B.J., A screw dislocation interacting with a coated fiber, Mech. Mater., 2000, 32(8), 485.

[13] Liu Y.W., Jiang C.P., Cheung Y.K., A screw dislocation interacting with an interphase layer between a circular inhomogeneity and the matrix, Int. J. Eng. Sci. 2003, 41(16),1883.

[14] Sudak L.J., Interaction between a Screw Dislocation and a ThreePhase Circular Inhomogeneity with Imperfect Interface, Math. Mech. Solids, 2003, 8(2), 171.

[15] Wang X., Pan E., Roy A.K., New phenomena concerning a screw dislocation interacting with two imperfect interfaces, J. Mech. Phys. Solids, 2007, 55(12), 2717.
[16] Honein E., Rai H., Najjar M.I., The material force acting on a screw dislocation in the presence of a multi-layered circular inclusion, Int. J. Solids Struct., 2006, 43(7), 2422.

[17] Miller R.E., Shenoy V.B., Size-dependent elastic properties of nanosized structural elements, Nanotechnology, 2000, 11(3), 139.

[18] Li X., Ono T., Wang Y., Esashi M., Ultrathin single-crystallinesilicon cantilever resonators: Fabrication technology and sig. nificant specimen size effect on Young's modulus, Appl. Phys. Lett., 2003, 83(15), 3081.

[19] Chang T., Gao H.J., Size-dependent elastic properties of a single-walled carbon nanotube via a molecular mechanics model, Mech. Phys. Solids, 2003, 51(6),1059.

[20] Zhu Y., Xu F., Qin Q., Fung W.Y., Lu W., Mechanical Properties of Vapor-Liquid-Solid Synthesized Silicon Nanowires, Nano Lett., 2009, 9(11), 3934.

[21] Sadeghian H., Goosen H., Bossche A., Thijsse B., Van Keulen F., On the size-dependent elasticity of silicon nanocantilevers: Impact of defects, J. Phys. D Appl. Phys., 2011, 44(7), 072001.

[22] Fleck N.A., Muller G.M., Ashby M.F., Hutchinson J.W., Train Gradient Plasticity: Theory and Experiment, Acta Metall. Mater., 1994, 42(2), 475.

[23] Fleck N.A., Hutchinson J.W., Strain Gradient Plasticity, Adv. Appl. Mech., 1997, 33, 295.

[24] Ma Q., Clarke D.R., Size dependent hardness of silver single crystals, J. Mater. Res., 1995, 10(4), 853.

[25] McElhaney K.W., Vlassak J.J., Nix W.D., Determination of indenter tip geometry and indentation contact area for depth-sensing indentation experiments, J. Mater. Res., 1998, 13(5), 1300.

[26] Xiang Y., Tsui T.Y., Vlassak J.J., The mechanical properties of freestanding electroplated Cu thin films , J. Mater. Res., 2006, 21(6), 1607.

[27] Nicola L., Xiang Y., Vlassak J.J., Van der Giessen E., Needleman A., Plastic deformation of freestanding thin films: Experiments and modeling, J. Mech. Phys. Solids, 2006, 54(10), 2089.

[28] Davoudi K.M., Nicola L., Vlassak J.J., Dislocation climb in twodimensional discrete dislocation dynamics, J. Appl. Phys., 2012, 111(10),103522.

[29] Davoudi K.M., Nicola L., Vlassak J.J., Bauschinger effect in thin metal films: Discrete dislocation dynamics study, J. Appl. Phys., 2014, 115(1), 13507.

[30] Davoudi K.M., Vlassak J.J., Dislocation Evolution During Plastic Deformation: Equations vs. Discrete Dislocation Simulations, J. Appl. Phys., 2018, 123(8), 085302.

[31] Aifantis E.C., Internal length gradient (ILG) material mechanics across scales and disciplines, Adv. Appl. Mech., 2016, 49, 1.

[32] Ru C.Q., Aifantis E.C., A simple approach to solve boundaryvalue problems in gradient elasticity, Acta Mech., 1993, 101(14), 59.

[33] Shodja H.M., Davoudi K.M., Gutkin M.Y., Analysis of displacement and strain fields of a screw dislocation in a nanowire using gradient elasticity theory, Scr. Mater., 2008, 59(3), 368.

[34] Davoudi K.M., Gutkin M.Y., Shodja H.M., Analysis of stress field of a screw dislocation inside an embedded nanowire using strain gradient elasticity, Scr. Mater., 2009, 61(4), 355.

[35] Davoudi K.M., Interaction between an edge dislocation near a circular void within the framework of the theory of strain gradient elasticity, J. Mech. Behav. Mater., 2018, 27(3-4). 